\section{The Bristol Oral Health Promotion Partnership}

\author{
By Eleanor Baldock, Benjamin Neo, Francesca Siodlak,
} Radhika Ladwa and Elorm (Julia) Atuwo



I $\mathrm{t}$ is astonishing that in 2019, the most significant contribution to paediatric general anaesthetic (GA) admissions is due to an almost entirely preventable disease.

In 2017-18 there were over 12,000 dental GA admissions for children under five, meaning $90 \%$ of GAs were due to dental causes ${ }^{1}$ This not only has implications for the general health of these children but also places an unnecessary strain on a health system that is already working under financial constraints. ${ }^{2}$

The UK is certainly placing more emphasis on a preventative dental health care system. Campaigns such as Dental Care by 1 (DCby1), ${ }^{3}$ initiated by the British Society of Paediatric Dentistry (BSPD), are excellent steps forward in targeting younger patients but despite this, $60 \%$ of children under five years old did not see a dentist in $2018 .{ }^{4}$ It is evident that we need to be doing more as a profession.

As newly qualified dentists on the Bristol and South Cotswolds Dental Foundation Training scheme we wanted to make a sustainable impact on our local community. Given the aforementioned statistics, we decided to target expectant and young mothers in association with the regional Health Visitors (HV) and Family Nurse Partnership (FNP). The FNP is a structured programme, partnering vulnerable young parents aged 24 and under with a specially trained family nurse. ${ }^{5}$ The programme aims to deliver tailored interventions targeted to the specific needs of these high risk families. We therefore found this programme an ideal platform to integrate oral health and selfcare, enabling young people to make the best decisions for themselves and their children.

In December 2018 we established the Bristol Oral Health Promotion Partnership (BOHPP), creating a collaboration between
Foundation Dentists and a variety of representatives from Bristol Children's services. The partnership was set up with the aim of improving the oral health of children in the Bristol area. Among other pursuits, the BOHPP aims to empower both HVs and the FNP with the knowledge they need to be able to deliver key oral health messages to new and expectant mothers. In doing this, we also hoped to have a positive impact on general health and wellbeing.

When meeting with representatives from Bristol Children's Services we found that $75 \%$ felt they had inadequate oral hygiene training and scored an average of $64 \%$ in the simple oral health quiz we asked them to complete. By using focus groups and workshops we were able to provide bespoke resources and interactive oral health tools for the nurses and their clients to aid learning and understanding. Both HVs and the FNP received training sessions from us focusing on oral hygiene instruction, diet advice and addressing common dental myths. Our hope is that with this new knowledge and resources provided, the child health professionals involved will feel more confident in delivering oral health advice to their clients and have a new appreciation for the role it can play in general health.

As a culmination of our year's work, we were invited to a young parents' event at We the Curious in Bristol. This event attracted over 350 families and gave us the opportunity to break down barriers between some of Bristol's most vulnerable families and the dental community. With sponsorship from GlaxoSmithKline we were able to provide families with free toothpastes, toothbrushes and oral health activity packs, whilst also engaging with parents and children to deliver key oral health messages using the interactive tools we had designed. As a result of this event the BOHPP officially signed up to the Bristol Children's Charter, a set of ten pledges made by organisations from across the city to set the rights and best interests of children as a priority for decision makers in Bristol.

Throughout our community project, we have received positive feedback from parents, health visitors and the family nurse partnership. Our resources are also being shared regionally between the Bristol, Bath, Swindon, Oxford and Wiltshire Family Nurses alongside being placed on their national website. We are also in the process of collaborating with contributors to the new electronic red book, a personal child health record provided to all children from the beginning of pregnancy until five years of age. Our aim is that our resources will be featured in the dental section of the e-redbook. Our ultimate goal is that our work this year provides a platform for a continued emphasis on the importance of oral health for vulnerable families within the South West area.

\section{References}

1. Public Health England. Hospital tooth extractions of 0 to 19 year olds. 2019. Online information available at https://www.gov.uk/government/publications/hospital-tooth-extractions-of-0-to-19-year-olds (accessed 2 September 2019)

2. Schneuer F J, Bentley J P, Davidson A J et al. The impact of general anesthesia on child development and school performance: a population-based study. Paediatr Anaesth 2018; 28: $528-536$.

3. Dental Check By One. 2017. https://dentalcheckbyone.co.uk (accessed 2 September 2019).

4. NHS Dental Statistics. Second Quarterly Report. 2019. Available online at https:// digital.nhs.uk/data-and-information/publications/statistical/nhs-dental-statistics/quarter-2-2018-19 (accessed 2 September 2019).

5. The Family Nurse Partnership. 2019. Available online at https://fnp.nhs.uk/about-us/ the-programme/ (accessed 2 September 2019). 